\title{
EXPERIMENTATION AND ADAPTIVE MODELING FOR TEMPERATURE EFFECT QUANTIFICATION IN PVP STRUCTURAL HEALTH MONITORING WITH PWAS
}

\author{
Tuncay Kamas \\ Eskisehir Osmangazi University \\ Eskisehir, Turkiye
}

\author{
Banibrata Poddar \\ University of South Carolina \\ Columbia, SC, USA
}

\author{
Bin Lin \\ University of South Carolina \\ Columbia, SC, USA
}

\author{
Lingyu Yu \\ University of South Carolina \\ Columbia, SC, USA
}

\author{
Victor Giurgiutiu \\ University of South Carolina \\ Columbia, SC, USA
}

\section{ABSTRACT}

The thermal effects at elevated temperatures mostly exist for pressure vessel and pipe (PVP) applications. The technologies for diagnosis and prognosis of PVP systems need to take the thermal effect into account and compensate it on sensing and monitoring of PVP structures. One of the extensively employed sensor technologies has been permanently installed piezoelectric wafer active sensor (PWAS) for in-situ continuous structural health monitoring (SHM). Using the transduction of ultrasonic elastic waves into voltage and vice versa, PWAS has been emerged as one of the major SHM sensing technologies. However, the dynamic characteristics of PWAS need to be explored prior its installation for in-situ SHM. Electro-mechanical impedance spectroscopy (EMIS) method has been utilized as a dynamic descriptor of PWAS and as a high frequency local modal sensing technique by applying standing waves to indicate the response of the PWAS resonator by determining the resonance and anti-resonance frequencies. Another SHM technology utilizing PWAS is guided wave propagation (GWP) as a far-field transient sensing technique by transducing the traveling guided ultrasonic waves (GUW) into substrate structure. The paper first presents EMIS method that qualifies and quantifies circular PWAS resonators under traction-free boundary condition and in an ambience with increasing temperature. The piezoelectric material degradation was investigated by introducing the temperature effects on the material parameters that are obtained from experimental observations as well as from related work in literature. GWP technique is also presented by inclusion of the thermal effects on the substrate material. The MATLAB GUI under the name of Wave Form Revealer (WFR) was adapted for prediction of the thermal effects on coupled guided waves and dynamic structural change in the substrate material at elevated temperature. The WFR software allows for the analysis of multimodal guided waves in the structure with affected material parameters in an ambience with elevated temperature.

\section{INTRODUCTION}

The thermal effects at elevated temperatures mostly exist for structural health monitoring (SHM) applications. The technologies for diagnosis and prognosis of SHM systems need to take the thermal effect into account and compensate it on sensing and in-situ monitoring of structures. One of the extensively employed sensor technologies has been permanently installed piezoelectric wafer active sensor (PWAS) for in-situ continuous SHM.

Piezoelectric wafer active sensor (PWAS) (Lin \& Giurgiutiu, 2010), (Giurgiutiu, Bao, \& Zhao, 2001) is lightweighted, inexpensive, unobtrusive, minimally intrusive sensor requiring low-power. PWAS is made of piezoelectric ceramic with electric field polarization, $E_{3}$, across the electrodes deposited on both surfaces. Using the transduction of ultrasonic elastic waves into voltage and vice versa, PWAS has been emerged as one of the major SHM sensing technologies and non-destructive evaluations (NDE) (Giurgiutiu, Zagrai, \& Bao, 2002; Giurgiutiu \& Zagrai, 2000). However, the dynamic characteristics of PWAS need to be explored prior its installation for in-situ SHM. Electro-mechanical impedance spectroscopy (EMIS) method has been utilized as a dynamic descriptor of PWAS and as a high frequency local modal sensing technique by applying standing waves to indicate the response of the PWAS resonator by determining the resonance and anti-resonance frequencies for in-situ ultrasonics (Zagrai \& Giurgiutiu, 2001) such as in the work presented by Liang et-al. 
(1994) and Sun et-al. (1994). They utilized the EMIS method for high frequency local modal sensing.

A literature survey is first conducted to assess prior work on the survivability of piezoelectric wafer active sensors (PWAS) at elevated temperature so that we could discover from the literature the extent of temperature dependence of the electric parameters, i.e. $d_{31}$ and $g_{31}$, and the elastic parameters, i.e. $s_{11}$ and Young's modulus $\left(c_{11}\right)$, of different piezoelectric materials.
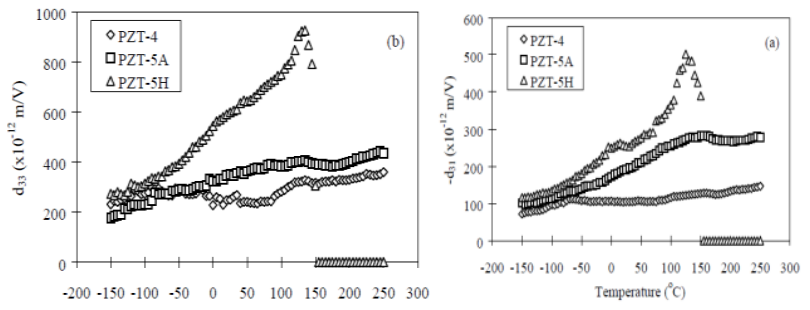

Figure 1 -d31 and d33 of three different PZT materials plotted as a function of temperature

A NASA report by (Hooker, 1998) shows the temperature dependence of $d_{31}$ and $d_{33}$, the effective E/M coupling coefficient as well as the thermal expansion of three different piezoelectric materials.

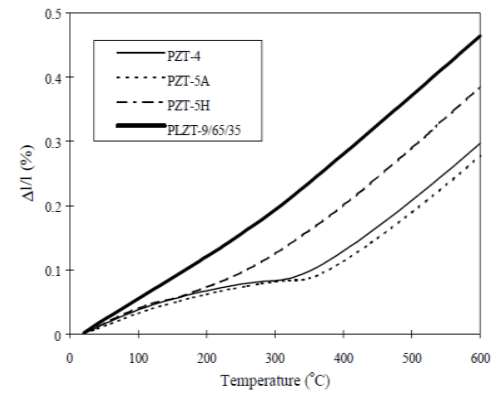

Figure 2 Thermal expansion of three different piezoelectric materials
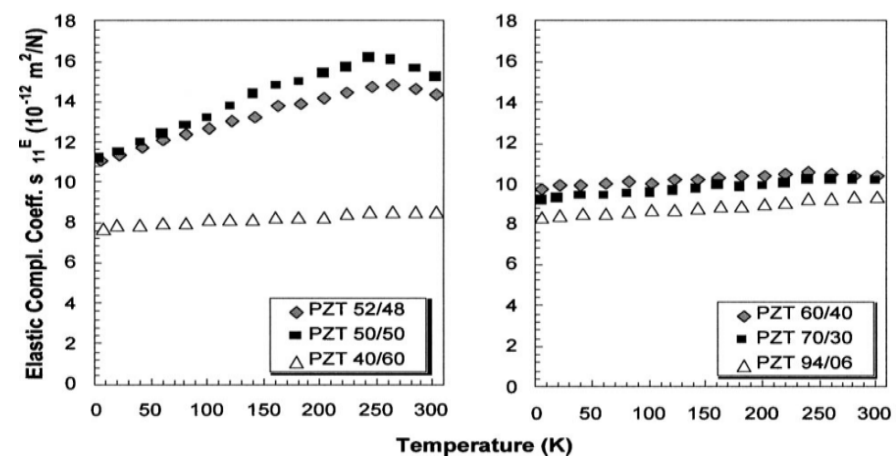

Figure 3 Low temperature elastic compliance coefficient (s11E ) plotted as a function of temperature for several tetragonal and rhombohedra PZT compositions.

Wolf (2004) reported increasing piezoelectric elastic compliance upto $250 \mathrm{~K}$ for PZT 52/48 and PZT 50/50 and their compliance values start decreasing after $250 \mathrm{~K}$ however other
PZT material with different $\mathrm{Zr} / \mathrm{Ti}$ compositions have monotonic increase until $300 \mathrm{~K}$ as seen in Figure 3.

Raghavan \& Cesnik in 2008 reported elastic and electric properties of a piezoelectric material, PZT-5A as a function of temperature raised up to $150{ }^{\circ} \mathrm{C}$ as seen in Figure 4. The inverse of Young's modulus, $\mathrm{Y}$, is the elastic compliance.
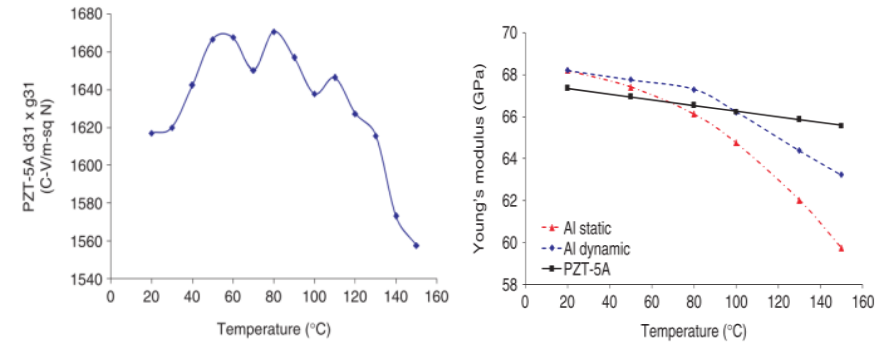

Figure 4 Variation of Young's modulus and $d_{31} * g_{31}$. Average thermal expansion for PZT-5A $\alpha_{\text {PZT }-5 \mathrm{~A}}=2.5 \mu \mathrm{m} / \mathrm{m}-{ }^{\circ} \mathrm{C}$.

Young's moduli of PZT-5A monotonically decrease as temperature increases between the room temperature and $160^{\circ} \mathrm{C}$. The product of $\mathrm{d}_{31} * \mathrm{~g}_{31}$ fluctuates along the temperature. It first monotonically inclines until $60{ }^{\circ} \mathrm{C}$, and it declines after $110{ }^{\circ} \mathrm{C}$ and it goes lower than its original value at room temperature.

Wave Form Revealer (WFR) is a software written in MATLAB GUI form developed by Shen (2014) at Laboratory for Active Materials and Smart Structures (LAMSS). The purpose of this software is to simulate Lamb wave propagation in plate structures made of various materials. But this software originally was not capable of simulating temperature effects on Lamb wave propagation.

This paper presents a theoretical and experimental study of the structural health monitoring capability of PWAS at elevated temperatures. Electro-mechanical impedance spectroscopy (EMIS) method is qualified using PWAS at increasing temperature. The material degradation is investigated by introducing the temperature effects on some parameters. The results from the analytical PWAS-EMIS simulations are presented. Temperature variation may produce pyro-electric charges, which may interfere with the piezoelectric effect. For the experimental validation, the EMIS measurements are conducted during the temperature increase. Then, the results from the experimental cycling of PWAS at gradually increasing temperatures are discussed. Trends of the results in terms of static capacitance, $\mathrm{C}_{0}$ and electromechanical impedance spectroscopy (EMIS) are presented.

Another goal of the paper was to develop software to simulate Lamb wave propagation in a specimen at variable temperature. This is required to understand the variability of several different substrate material properties with temperature and how this variability effects the Lamb wave propagation in substrate structures. This preliminary study was conducted for plates however could be extended to cylindrical structures such as pressure vessels and pipes. 


\section{EMIS SIMULATIONS FOR PWAS AT ELEVATED TEMPERATURES}

In this subsection, the temperature effects on free circular PWAS admittance and impedance are presented through the analytical model and the EMIS tests. The effects of the stiffness coefficient $\mathrm{c}_{11}$, the piezoelectric coefficient $\mathrm{d}_{31}$, and the static capacitance $\mathrm{C}_{0}$ on impedance/admittance are taken into account.

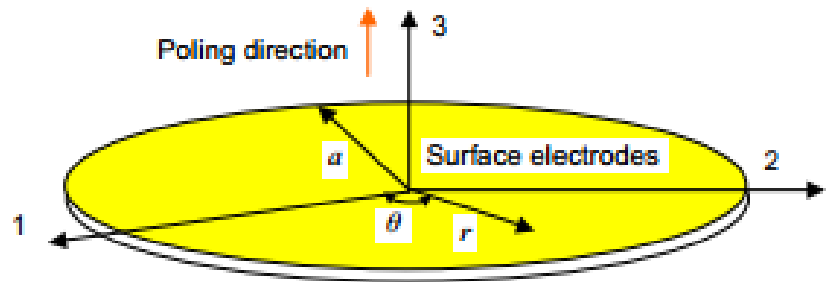

Figure 5 Schema of circular PWAS in cylindrical coordinate system Zagrai (2002).

Zagrai (2002) has developed 2-D EMIS for circular PWAS using the free circular PWAS model (Figure 5) and the derivation procedure shown in flow-chart in paper authored by Kamas et-al. (2015). In this section, we adopted herein in-plane EMIS model developed by Zagrai to simulate the temperature effects on piezoelectric material degradation of free circular PWAS. The analytical simulation will be conducted by changing the stiffness coefficient, the piezoelectric coefficient, and the capacitance. The stiffness coefficient and the piezoelectric coefficient degradation have been discussed in the literature and plots for the material properties versus temperature increase have been provided. The capacitance dependence over temperature has been defined during our experimental studies. Therefore, the proportions of the elastic and piezoelectric material property degradations are attained from the literature and the capacitance proportion was obtained from the capacitance measurements over increasing temperature. The admittance and impedance simulations are presented respectively and compared with the experimental results.

\section{EXPERIMENTAL WORK}

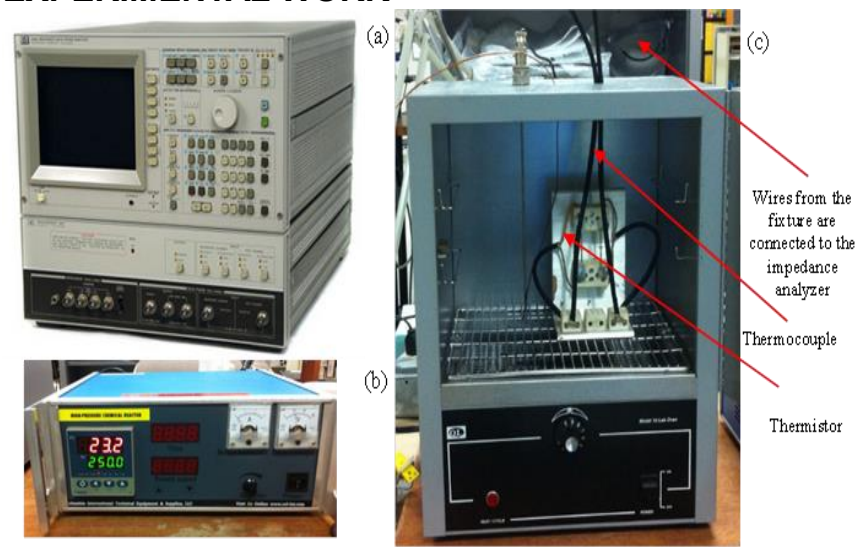

Figure 6 High temperature PWAS testing by (a) impedance analyzer, (b) PID temperature controller (c) oven.

The E/M impedance is used as a direct and convenient method to implement for PWAS impedance signature as a function of temperature up to relatively high temperature, the required equipment being an electrical impedance analyzer, such as HP 4194A impedance analyzer, PID temperature controller, and oven. An example of performing PWAS E/M impedance spectroscopy is presented for PWAS located in a fixture in the oven in Figure 6. PWAS has to have stress-free i.e. unconstrained boundary conditions so that it was fixed by pogopins that only apply low spring forces point-wise on the PWAS surfaces in the fixture. The fixture has wires that can be connected with the probes of the EMIS analyzer instrument. The impedance analyzer reads the E/M impedance of PWAS itself in the oven. It is applied by scanning a predetermined frequency range $(300 \mathrm{kHz}-400 \mathrm{kHz})$ and recording the complex impedance spectrum. A LabView data acquisition program was used to control the impedance analyzer and sweep the frequency range in steps (of $100 \mathrm{~Hz}$ ) that was predefined and to attain the data in a format that assists to data analysis. During the visualization of the frequency sweep, the real part of the E/M impedance, $\operatorname{Re}(Z(\omega))$, follows up and down variation as the structural impedance goes through the peaks and valleys of the structural resonances and anti-resonances.

\section{RESULTS AND DISCUSSION}

In this subsection, we present admittance results from free circular PWAS resonators at elevated temperatures using the same experimental setup. In Figure 7, 3-D plots of the admittance in frequency domain over time are illustrated for the measurements conducted at different temperatures. The temperature values for the each admittance measurement were kept constant by the closed loop temperature controller and monitored and recorded by the GUI software that was specifically designed and created by Jingjing (Jack) Bao and Bin Lin in LAMSS using LabVIEW for this admittance tests. 
Admittance at elevated temperature between RT and $50^{\circ} \mathrm{C}$

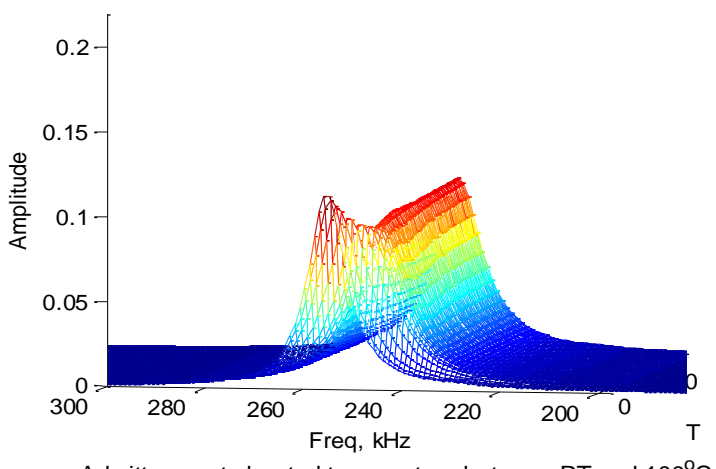

Admittance at elevated temperature between RT and $100^{\circ} \mathrm{C}$

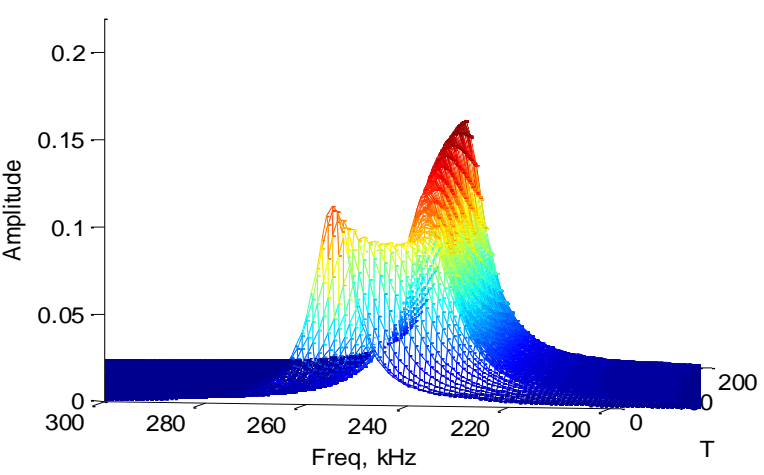

Admittance at elevated temperature between RT and $150^{\circ} \mathrm{C}$

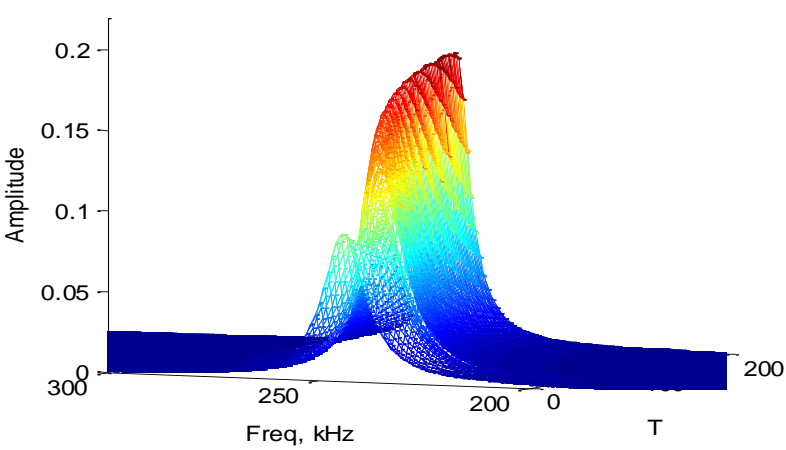

Admittance at elevated temperature between RT and $200^{\circ} \mathrm{C}$

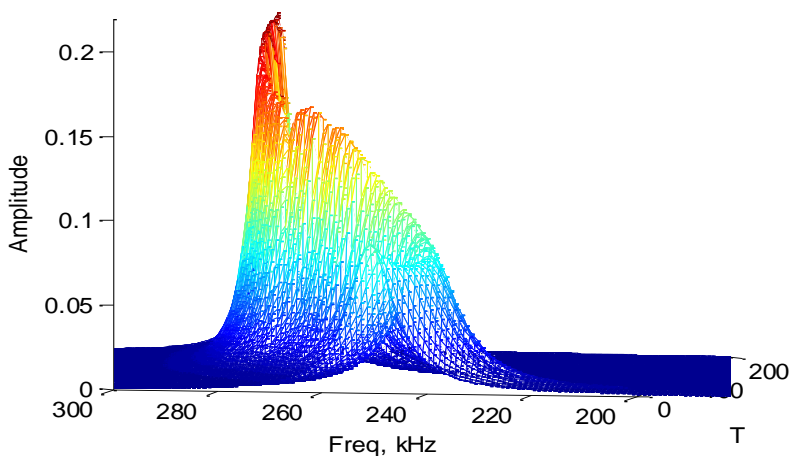

Figure $73 \mathrm{D}$ contour plots for admittance spectra at elevated temperatures.

Admittance amplitudes from a PWAS have been measured during the temperature increases from the room temperature to the elevated temperatures and the results were plotted and presented in Figure 8. The admittance trend inclines as the temperature increases with the similar trend from the room temperature toward the elevated temperatures. Even though the admittance amplitude recovers after the PWAS cools down to the room temperature from the $50^{\circ} \mathrm{C}$, it cannot recover and drops down in the amplitude after the PWAS cools down from the higher temperatures. Another interesting behavior that can be observed in this test results is that the amplitude keeps increasing although the temperature remains the same at the elevated temperatures.

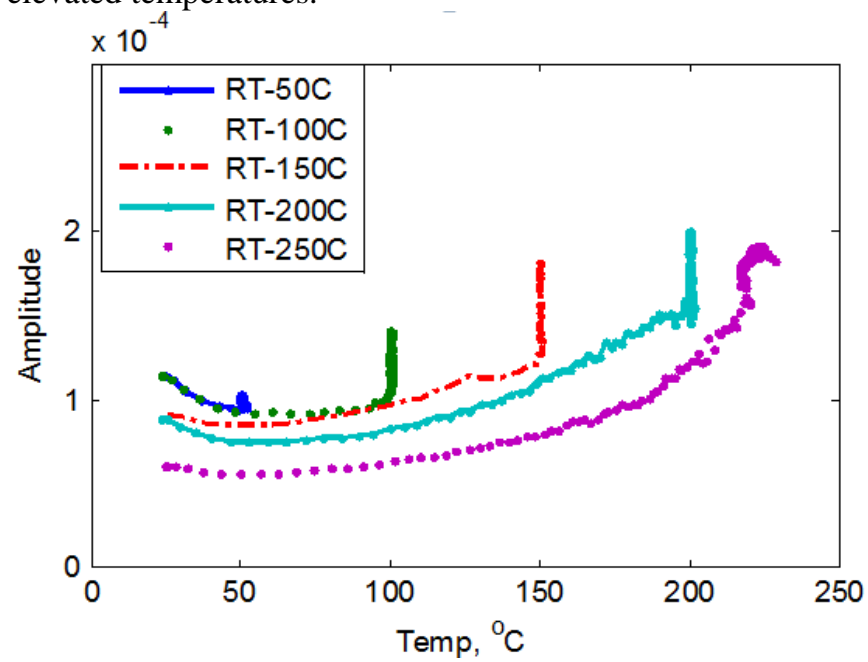

Figure 8 Admittance peak amplitude at the first in-plane resonance frequency of circular PWAS at elevated temperatures.

The first in-plane resonance frequency values of free PWAS in an oven as the temperature was increasing are plotted as shown in Figure 9. The similar phenomena can be observed in the frequency shifts as seen in the amplitude shifts.

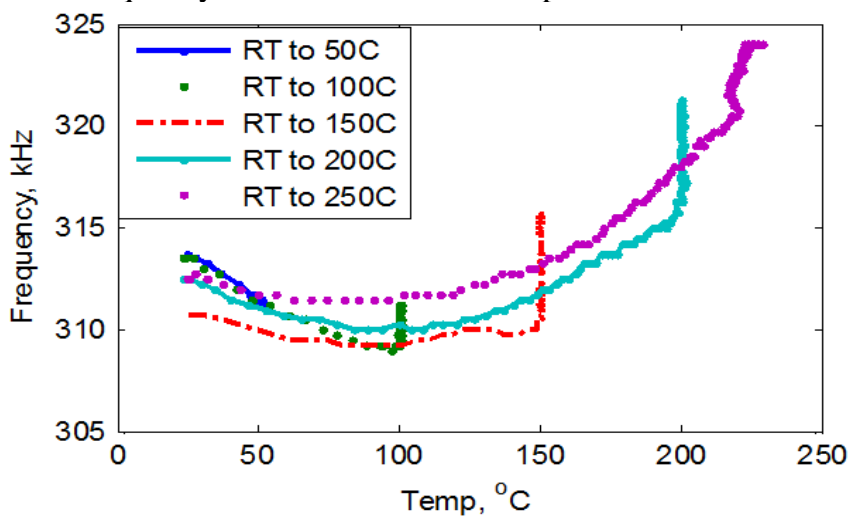

Figure 9 Admittance frequency at the first in-plane resonance frequency of circular PWAS at elevated temperatures. 

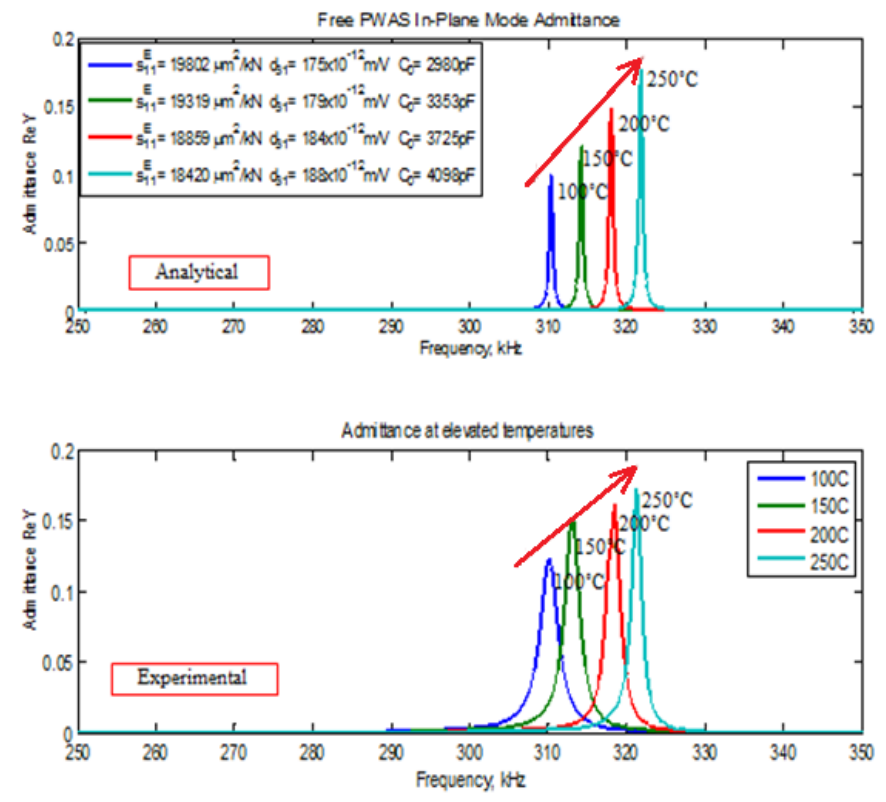

\section{SIMULATIONS OF TEMPERATURE EFFECTS ON GUIDED WAVE PROPAGATION}

For simulating the effects of elevated temperature on the Lamb wave propagation we have changed the user input to WFR from material properties of the plate to the temperature of the plate (Figure 10). This was required since all the material properties of the plate depend on the temperature of the plate. Therefore if we know the temperature we can estimate the material properties internally within WFR using experimental data of the temperature dependence of the material properties.

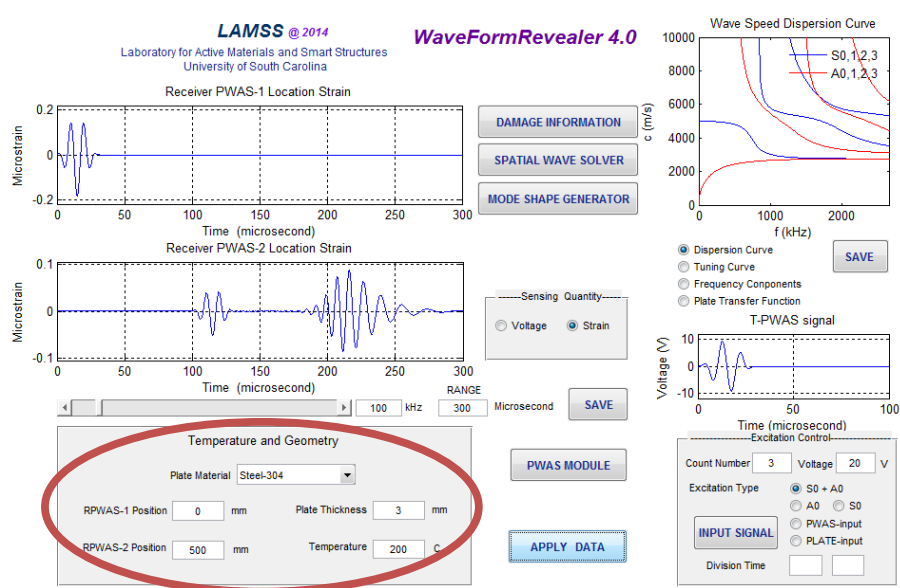

Figure 10 Wave Form Revealer (new version)

The temperature dependent material property data was obtained from literature and other web resources. The resources are mentioned in the reference. WFR interpolates stored material property data to predict the material property at the user specified temperature. This facilitates us to use WFR for simulating Lamb wave propagation at variable temperature.
We want to use the effect of temperature variation on each of the material properties $(E, \rho, v)$ separately and analyze its effect on Lamb wave propagation. Presently we used only the variation of elastic modulus with temperature to capture the effects of temperature on Lamb wave propagation. In Figure 11, we can see that the experimental data shows the variation of elastic modulus of steel 304 with temperature at specific temperatures. For a given temperature the software uses this stored data and interpolates it to get the estimated elastic modulus. This way many other material prperties can be interpolated and used for Lamb wave simulatation at elevated temperatures.

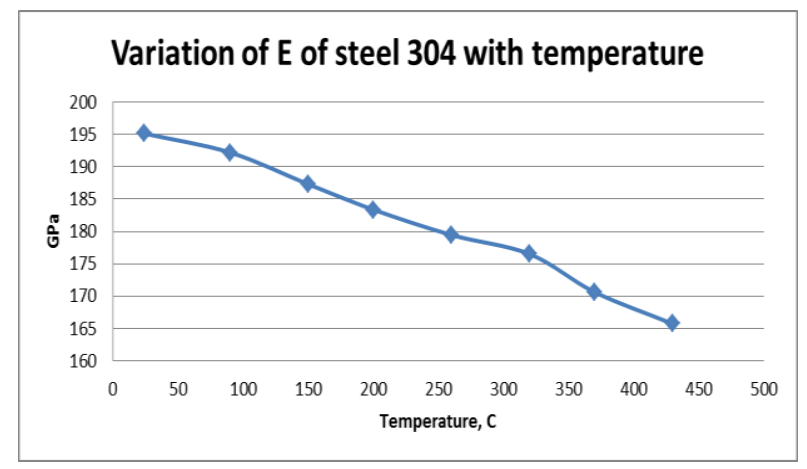

Figure 11 Experimental data of material property

For determining the material properties at the given temperature the softare relies on stored experimental data.

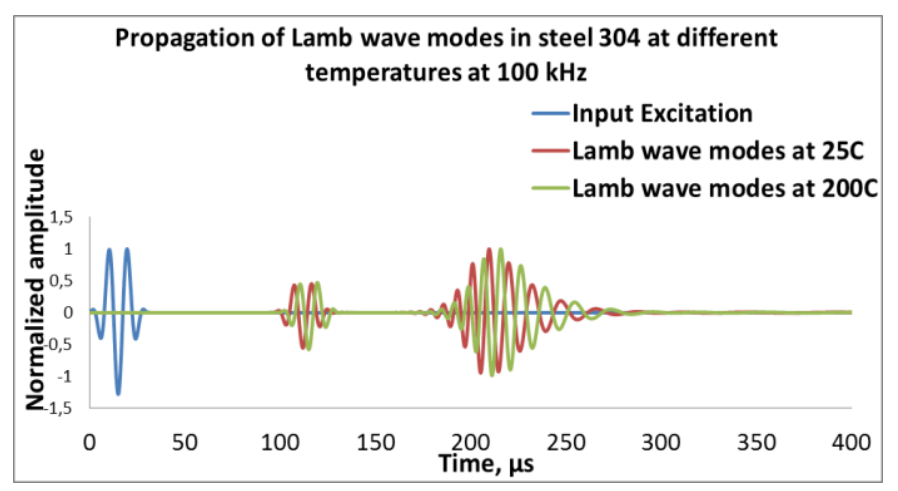

Figure 12 Lamb wave modes simulated by WFR new version with temperature input at $25^{\circ} \mathrm{C}$ and $200^{\circ} \mathrm{C}$

In Figure 12, even though the input excitation is the same for the Lamb wave modes in steel, we can observe a phase shift as the temperature increased. The first Lamb wave mode is the S0 wave packet and the second is the A0 mode. As seen, the phase shift in A0 wave packet is more in comparison to that in the S0 mode. 


\section{SUMMARY AND CONCLUSIONS}

Two SHM technologies have been utilized in this work: one is the electro-mechanical impedance spectroscopy (EMIS) method. We utilized it as a dynamic descriptor of PWAS and as a high frequency local modal sensing technique by applying standing waves to indicate the response of the PWAS resonator by determining the resonance and anti-resonance frequencies. Another SHM technology utilizing PWAS generated wave propagation (GWP) as a far-field transient sensing technique by transducing the traveling guided ultrasonic waves (GUW) into substrate structure. The paper first presents EMIS method that qualifies and quantifies circular PWAS resonators under traction-free boundary condition and in an ambience with increasing temperature. The piezoelectric material degradation was investigated by introducing the temperature effects on the material parameters that are obtained from experimental observations as well as from related work in literature. Second, GWP technique is presented by inclusion of the thermal effects on the substrate material. The MATLAB GUI under the name of Wave Form Revealer (WFR) was adapted for prediction of the thermal effects on coupled guided waves and dynamic structural change in the substrate material at elevated temperature. The WFR software allows for the analysis of multimodal guided waves in the structure with affected material parameters in an ambience with elevated temperature.

\section{ACKNOWLEDGMENTS}

The following financial support agencies are thankfully acknowledged: National Science Foundation Grant \# CMS0925466; Office of Naval Research \# N00014-11-1-0271, Dr. Ignacio Perez, Technical Representative; Air Force Office of Scientific Research \#FA9550-11-1-0133, Dr. David Stargel, Program Manager; Nuclear Energy University Program Grant \# DE-NE0000726, Kenny Osborne, Project Manager.

\section{REFERENCES}

Giurgiutiu, V., Bao, J., \& Zhao, W. (2001). Active Sensor Wave Propagation Health Monitoring of Beam and Plate Structures. In Proc of SPIE's 8th International Symposium on Smart Structures and Materials. Newport Beach, CA.

Giurgiutiu, V., Zagrai, a., \& Jing Bao, J. (2002). Piezoelectric Wafer Embedded Active Sensors for Aging Aircraft Structural Health Monitoring. Structural Health Monitoring, 1(1), 41-61. doi:10.1177/147592170200100104

Giurgiutiu, V., \& Zagrai, A. (2000). Damage Detection in Simulated Aging-Aircraft Panels Using The ElectroMechanical Impedance Technique. In Adaptive Structures and Material Systems Symposium, ASME Winter Annual Meeting (pp. 1-10). Orlando, FL.
Hooker, M. W. (1998). Properties of PZT-Based Piezoelectric Ceramics Between - 150 and 250 C. Hampton, Virginia.

Kamas, T., Frankforter, E., Lin, B., Yu, L. (Lucy), \& Giurgiutiu, V. (2015). Thermal Effect on E/M Impedance Spectroscopy of Piezoelectric Wafer Active Sensors. In SPIE 2015 Smart Structure/NDE (pp. 1-10). San Diego, CA.

Liang, C., Sun, F. P., \& Rogers, C. a. (1994). Coupled ElectroMechanical Analysis of Adaptive Material Systems -Determination of the Actuator Power Consumption and System Energy Transfer. Journal of Intelligent Material Systems and Structures, 5(1), 12-20. doi:10.1177/1045389X9400500102

Lin, B., \& Giurgiutiu, V. (2010). Modeling of Power and Energy Transduction of Embedded Piezoelectric Wafer Active Sensors for Structural Health Monitoring, 7981, 76472P-76472P-12. doi:10.1117/12.880120

Raghavan, a., \& Cesnik, C. E. S. (2008). Effects of Elevated Temperature on Guided-wave Structural Health Monitoring. Journal of Intelligent Material Systems and Structures, 19(12), 1383-1398. doi:10.1177/1045389X07086691

Shen, Y. (2014). Structural Health Monitoring Using Linear and Nonlinear Ultrasonic Guided Waves. University of South Carolina.

Sun, F. P., Liang, C., \& Rogers, C. A. (1994). Structural modal analysis using collocated piezoelectric actuator/sensors: an electromechanical approach. In Proc. SPIE 2190, Smart Structures and Materials 1994: Smart Structures and Intelligent Systems, 238. Orlando, FL.

Wolf, R. a. (2004). Temperature dependence of the piezoelectric response in lead zirconate titanate films. Journal of Applied Physics, 95(3), 1397. doi:10.1063/1.1636530

Zagrai, A. N. (2002). Piezoelectric Wafer Active Sensor Electro-Mechanical Impedance Structural Health Monitoring. University of South Carolina. Retrieved from http://onlinelibrary.wiley.com/doi/10.1002/cbdv.2004901 37/abstract

Zagrai, A. N., \& Giurgiutiu, V. (2001). Electro-Mechanical Impedance Method for Damage Identification in Circular Plates, 40. 